\title{
Understanding, evaluating, and supporting self-regulated learning using learning analytics
}

\author{
Ido Roll \\ University of British Columbia, Canada \\ ido.roll@ubc.ca \\ Philip H. Winne \\ Simon Fraser University, Canada
}

\begin{abstract}
Self-regulated learning is an ongoing process rather than a single snapshot in time. Naturally, the field of learning analytics, focusing on interactions and learning trajectories, offers exciting opportunities for analyzing and supporting self-regulated learning. This special section highlights the current state of research at the intersection of self-regulated learning and learning analytics, bridging communities, disciplines, and schools of thought. In this opening article, we introduce the papers and identify themes and challenges in understanding and supporting selfregulated learning in interactive learning environments.
\end{abstract}

KEYWORDS: self-regulated learning, learning analytics

\section{INTRODUCTION}

In line with many models of self-regulated learning and, more generally, constructivism, we adopt the view that learners are agents (Jonassen, 1991). Agents seek out, choose and carry out options to achieve goals. However, whether by design or not, a learner's environment constrains the possibility of achieving particular goals and of taking particular steps to approach a goal. Experimenters and instructors may interpret that their interventions directly change processes learners use in learning, what learners learn, and learners' motivations and affective stances. But, they are mistaken. Interventions do not determine how learners engage with tasks. Rather, interventions are affordances that agentic learners meld with other elements in the context as they perceive it to regulate learning.

In terms of one widely cited model of self-regulated learning proposed by Winne and Hadwin (1998; Winne, 2011; Greene \& Azevedo, 2007), learners exercise agency across four loosely sequenced phases: (1) They scan their environment to identify internal factors (cognitive, motivational, affective) and external features that may influence a task. (2) They frame goals and design plans to approach them. (3) They implement actions to animate their plans, monitor the match between a plan and its actualization and modestly adjust actions as they judge appropriate. And, (4) they re-examine aspects across these three prior phases to consider major, strategic revisions to understanding and action if progress toward goals is blocked, too slow or in some other way unsatisfactory. From this perspective, agentic learners 
are inherently self-regulating learners. Learners regulate their learning constantly, even if not always successfully as judged by the learner or by another, e.g., a teacher or researcher.

While the importance of self-regulated learning (SRL) to learning is obvious (Bransford, 2000), understanding how SRL is applied in context is not a simple task. We know how to interpret and evaluate a learner's knowledge of domains in terms of correctness or the quality of solutions to problems. Yet interpreting and evaluating qualities of actions, strategies, goals and, more broadly, regulation is a much more challenging task (Roll et al., 2014). Data gathered in online learning environments are instrumental to addressing this challenge. However, without appropriate tools, theories, and frameworks, data alone are insufficient to shape theory and guide practice. In this context, learning analytics can play an immensely important role for learners and for the learning sciences.

Learning analytics are reports of analyses of data that describe features of, and factors that influence, SRL. At the intersection of learning analytics and SRL, researchers can realize significant opportunity to better research and understand learning in authentic learning settings. Because learners are agentic, learning analytics can inform them about options that may bear on the phases of their self-regulated learning as described in Winne and Hadwin's model. Finally, expanding our understanding how SRL unfolds in context spurs developing learning environments that are concurrently better tools for gathering data for learning analytics and better at helping learners learn.

To realize the potential learning analytics for advancing the learning sciences, we collectively need to undertake three main kinds of research. The first relates to developing instrumentation to record traces of self-regulated learning across all its phases. Without data, neither learners nor learning scientists can progress toward their respective goals. Second, methods need to be fashioned that identify structures within data. Correspondingly, methods need to be tested for the extent and conditions they contribute to valid inferences about the constructs that the structures of data represent (Roll, Baker, Aleven, \& Koedinger, 2014). Thirdly, standing on these pillars of instrumentation (data collection) and methodologies that operate on data, learners and learning scientists alike are positioned to explore the effects of interventions. We construe interventions broadly as changes in a learning environment that range over internal cognitive, motivational and affective states of a learner as well as information externally introduced to learners by experimenters instructors or peers. In keeping with our axiom that learners are agents, we characterize interventions as affording opportunities for learners to more productively regulate learning in the service of achieving outcomes they value.

\section{ABOUT THE SPECIAL SECTION}

This special section showcases current exciting opportunities for learning analytics and learning sciences. Data and computing power are no longer a bottleneck. It is now time to re-examine theories, methods, and practice, hand-in-hand, promoting a synergy among these three pillars. The research reported in this special section illustrates important steps made in each of these areas of work. The first paper by Segedy, Kinnebrew, and Biswas (this special section) introduce Coherence Analysis - an analytical framework for interpreting students' actions in an exploratory learning environment. 
Coherence analysis interprets sequences of actions, and evaluates whether learners make use of information they collect. Thus, coherence analysis offers a tool to evaluate learning behaviours that are highly contextualized. The second paper, by Cutumisu, Blair, Chin, and Schwartz (this special section) also focus on assessing SRL, albeit using a different approach. Cutumisu and colleagues introduce Posterlet, a learning environment designed to evaluate students' tendencies to seek actionable feedback and act upon it. In addition to offering this environment, Cutumisu and colleagues shed some light on skills comprising SRL and other learner attributes. Whereas their paper evaluates the impact of feedback at the domain level, Sonnenberg and Bannert, the third paper in this special section, take a different approach by focusing on the effect of metacognitive prompting. They offer a broad analysis of their intervention using verbal protocol analysis, trace data analysis, and assessment of learning.

Two additional papers are included in a Work in Progress part of this special section. Nussbaumer, Hillemann, Gütl, and Albert take a theory-driven approach to design and implement an architecture for supporting students' SRL in a variety of activities. Their architecture is interesting in that it builds on multiple approaches to SRL, and combines intelligent models with open learner model. Colthorpe, Zimbardi, Ainscough, and Anderson look for traces of SRL and metacognition in students' use of a common environment, the Learning Management System. By comparing students' traces, self-reports, and academic performance, their work adds to our understanding of SRL in academic online settings.

The diversity of papers in this special section offers an interesting snapshot of the current state of research into SRL and learning analytics. Several themes emerge:

- Choices. Rather than looking at the content of learners' actions, we can look at the choices that learners make. SRL theories describe how students manage their learning, and open ended environments that gather trace data about these processes allow us to evaluate the types of actions students choose to perform, rather than only their content (Cutumisu; Colthorpe)

- Relativity. Actions are neither inherently good nor bad. Rather, choices students make reflect their perceived challenges, knowledge, prior experiences, and habits. Thus, actions should be interpreted in relation to a learner's context (Biswas; Nussbaumer).

- Reusable analytics. The volume of learning data gathered in online settings affords new opportunities to explore complex research questions. New methods and analytical techniques that provide insight into the complexity of SRL represented in these spaces are required. Specifically, we see a shift from looking at individual events to looking at sequences of actions (Biswas; Bannert). Developing new tools that can be reused across contexts and settings can contribute to the generality of theories and to support and evaluate transfer of SRL patterns (Biswas; Colthorpe).

- Challenge: operationalization. Many of the papers address similar constructs (e.g., planning, revising) and operationalize them in different ways, contextualized in their respective systems. Learning analytics allows us to take abstract constructs and instantiate them in different learning environments. Comparing and contrasting these instantiations can help us evaluate the effect of contextual and environmental factors on learners' SRL. 


\section{FUTURE DIRECTIONS FOR RESEARCH}

The sum of papers in this special section also highlights opportunities for future research. In a general sense, learning to self-regulate involves identifying patterns for later use. Patterns can offer greater information value that may improve the predictability of goal attainment and enhance a learner's understanding (theory) about which actions relate to goal attainment. The world, however, is stochastic; so methods for detecting patterns - human and machine - must wrestle with noise and indeterminacy.

One approach to this problem requires two distinct moves. The first is gathering an increased variety of types of data such that each type of data is relatively statistically independent of other types. The second move is to massively increase the volume of data. Elevating dimensionality of data in these two ways can mitigate two problems. First, adding dimensionality to data combats randomness expressed in the form of ignorance about factors that play causal roles. Second, worries about overfitting models can be lessened because methods that cluster or partition vastly dimensional, big data can be applied to form relatively homogeneous subsets in which variance is less prevalent than it is in the complete pool. Together, these two tacks are merely a post hoc operationalization of the lab experimenter's everyday stance toward controlling a potentially confounding factor in an empirical study.

This two-pronged approach leads to four implications for learning analytics and research on selfregulated learning. First, instrumentation needs to be designed that learners can use nearly ubiquitously. This helps to increase the volume of data. Winne and his colleagues' nStudy platform (e.g., Winne, 2015) and content-agnostic learning management systems illustrate this step. However, as learning technologies evolve, the footprint of any single system shrinks. Thus, systems need to support better interoperability. New technologies and standards (such as TinCan XAPI) attempt to bridge this gap. Second, algorithms capable of detecting partitions or clusters in vastly dimensional, big data need to be improved or invented. Presently, analytics that are supposedly general are often tested only in one setting. Researchers should evaluate the generalizability not only of theories, but also of tools and methodologies. Third, as data are collected more ubiquitously, we need to do a better job of attending to and capturing features of context. While systems collect information about students' actions, it is rare that enough data are available to describe both static and modulating external factors such as learner and task characteristics. Interpreting analytics about SRL needs to take account of the context in which data were collected. Last, new research is needed that investigates how to meaningfully convey analytics to learners when the data analyzed originates in vastly dimensional data. The challenge here is to explore how options for self-regulating learning are usefully described given the likely complexity of patterns of learning activities. In other words, how can information overload be avoided while not stripping out necessary information about how learning activities unfold over time?

Future research on learning analytics in service of understanding and promoting productive selfregulated learning should also tackle challenges presented by the very probable, and sometimes actively encouraged, condition that learning is social. One attempt to sidestep this challenge is to entertain a view that interactions among learners are no different than interactions a focal learner has with a static text. On a turn-by-turn basis, this is reasonable. Like Zeno's apparent paradox of Achilles and the 
tortoise (wherein Achilles never catches a tortoise allowed a head-start because, in each successive interval of time, Achilles covers only a portion of distance the has travelled in that time interval), the turn-by-turn view is inadequate. A genuinely social exchange consists of successive turns are not independent (not Markovian).

Wait - isn't this temporal structure fundamentally the same as temporally unfolding SRL wherein an agentic learner reviews her learning history to judge and adjust learning activities? Yes, it is. We surmise a bridge to join the solo to the social context for regulated learning activities lies in using the topic a learner studies as a means to follow how "threads" of content unfold across time in conjunction with patterns of self-regulated learning. To our knowledge, methods are not yet available that can simultaneously track patterns in which elements simultaneously represent the topic (subject matter, features of group process, a learner's affect) in the same unit as a representation of the cognitive operations applied to that topic. We recommend this as a topic worthy of future work. Furthermore, as learning is often a social process, designs for learning analytics need to take account of how a group regulates its learning, i.e., forms of co-regulated and socially-shared regulation of learning (Hadwin, Jarvella, and Miller, 2011). The field needs to make progress toward mapping and developing learning analytics for nested models of regulated learning.

Finally, many of the challenges faced by learning analysts who explore self-regulated learning are shared with colleagues working in the field of data mining (see Winne \& Baker, 2013). Strengthening bridges that join the learning analytics and data mining communities will benefit and, perhaps, even merge these two fields of work. We recommend scholars and funders find ways to promote increased exchanges amongst members of these communities.

Overall, the intersect of learning analytics and SRL offers a grand challenge. Grand in its magnitude; grand in its potential impact; and grand in that opportunities for meaningful progress are within reach. This special section takes several significant steps along the path to address this challenge.

\section{REFERENCES}

Bransford, J. D., Brown, A. L., \& Cocking, R. R. (2000). How people learn: Brain, mind, experience, and school. Washington, D.C.: National Academy Press.

Greene, J. A., \& Azevedo, R. (2007). A theoretical review of Winne and Hadwin's model of self-regulated learning: New perspectives and directions. Review of Educational Research, 77(3), 334-372.

Hadwin, A. F., Järvelä, S., \& Miller, M. (2011). Self-regulated, co-regulated, and socially shared regulation of learning. In D. H. Schunk \& B. Zimmerman (Eds.), Handbook of self-regulation of learning and performance (pp. 65-84). New York: Routledge.

Jonassen, D. H. (1991). Objectivism versus constructivism: Do we need a new philosophical paradigm?. Educational Technology Research and Development, 39(3), 5-14.

Roll, I., Wiese, E., Long, Y., Aleven, V., \& Koedinger, K. R. (2014). Tutoring self- and co-regulation with intelligent tutoring systems to help students acquire better learning skills. In R. Sottilare, $A$. Graesser, X. Hu, \& B. Goldberg (Eds.), Design Recommendations for Adaptive Intelligent Tutoring 
(2015). Understanding, evaluating, and supporting self-regulated learning using learning analytics. Journal of Learning Analytics, 2(1), 7-12.

Systems: Volume 2 - Adaptive Instructional Strategies (pp. 169-182). Orlando, FL: U.S. Army Research Laboratory.

Roll, I., Baker, R.S.J.d., Aleven, V., \& Koedinger, K. R. (2014). On the benefits of seeking (and avoiding) help in online problem solving environment. Journal of the Learning Sciences, 23:4, 537-560, doi: $10.1080 / 10508406.2014 .883977$

Winne, P. H. (2011). A cognitive and metacognitive analysis of self-regulated learning. In B. J. Zimmerman and D. H. Schunk (Eds.), Handbook of self-regulation of learning and performance (pp. 15-32). New York: Routledge.

Winne, P. H. (in press, 2015). Leveraging big data to help each learner upgrade learning and accelerate learning science. Teachers College Record, 117(9).

Winne, P. H., \& Hadwin, A. F. (1998). Studying as self-regulated learning. In D. J. Hacker, J. Dunlosky, \& A. C. Graesser (Eds.), Metacognition in educational theory and practice (pp. 277-304). Mahwah, NJ: Lawrence Erlbaum Associates. 